\title{
CME quiz discussion
}

These discussions relate to the June 1987 JAOA CME quiz.

1. (c). Indium-1ll labeled leukocyte scintigraphy is an accurate, noninvasive test for the detection of abscesses, especially acute processes.

2. (d). Indium-lll has a half-life of 67 hours, emits two gamma photons of $173 \mathrm{kev}$ and $247 \mathrm{kev}$, and does not alter cell viability in the concentration used for leukocyte scintigraphy.

3. (e). Periorbital swelling, decreased visual acuity, cranial bruit, and basal skull fracture are all commonly associated with post-traumatic cavernous-carotid fistulae.

4. (b) The diagnosis of cavernous carotid fistula can be made with certainty with CT.

5. (b) Most of the patients in the series reported by Kelch and Nowak and others in the literature had normal prothrombin and partial thromboplastin times.

6. (b) A review of cases in the literature indicates that delivery of the infant usually reversed the course of the patient's disease. Heparin has had equivocal results and may be associated with risks in a patient population in which the rate of cesarean section is high. Pritchard and associates found steroids of little value in these cases.

7. (b) The patient who is suffering from a chronic illness is confronted with a multiple of losses. The easiest to identify for both the patient and the physician are the real losses. However, symbolic loss and fantasized loss, despite being much more difficult to identify, usually evoke a more severe response. Symbolic and fastasy losses are often centered around the same event. It should be kept in mind that fantasized losses can become real losses.

8. (b) Regression is one of the most common emotional responses to illness. Passivity and dependency, associated with the onset of illness and its treatment, can lead to a regression to a psychologic state appropriate to a time in the patient's life when dependency was acceptedchildhood. Regression can become detrimental if it becomes too severe or if it goes on too long. 